\title{
Stereotactic Neurosurgery Planning on a Personal-Computer-Based Work Station
}

\author{
T.M. Peters, J.A. Clark, G.B. Pike, C. Henri, L. Collins, D. Leksell, and O. Jeppsson
}

\begin{abstract}
Stereotactic surgery requires knowledge of cerebral structures derived from more than one image source. We have developed a PC-AT-based workstation that accepts patient images, made with the stereotactic frame in place, from CT, MRI, and DSA modalities. Reference markers on the frame are identified in the images to establish the coordinate geometry for each modality. Target points may be identified on each image type and trajectories of probe paths to these points defined. Targets identified on one set of images may be transferred automatically to other images of the same patient in order to guarantee a vessel-free path of approach to a target point deep within the brain. To date several hundred patients have had stereotactic surgery performed on the basis of plans using this system. Procedures included biopsy and aspiration of lesions, implantation of electrodes for the recording of deep EEG signals, and radiosurgical techniques. We present clinical examples of the use of this system in typical stereotactic neurosurgery procedures. address stereoscopic applications, and discuss the results of intermodality tests to establish the accuracy of the technique. (C) 1989 by W.B. Saunders Company.
\end{abstract}

$\mathbf{S}$ TEREOTACTIC surgery is a means whereby lesions deep within the brain may be approached either by way of a small twist-drill hole in the skull or by an external narrow beam of high-energy ionizing radiation. ${ }^{1-3}$ Before any of these procedures can be attempted, the precise locations of the "target"points (with respect to some fixed reference) within the brain must be accurately determined. To do this, a reference frame is rigidly fixed to the patient's head with carbon-fiber or aluminum pins that fit snugly into shallow recesses drilled in the skull, and is left in place during the imaging process. This frame contains a scale from which the coordinates of points inside the frame may be measured, and also a set of reference (or fiducial) markers whose positions are accurately known with respect to the frame and which may be easily identified on the images. ${ }^{4}$

Imaging modalities used for this purpose include computed tomography (CT), digital subtraction angiography (DSA), and magnetic resonance imaging (MRI). While there are a number of stereotactic frames available, ${ }^{1,4,5}$ we describe one that embodies the common features of most of them. The unit used at the Montreal Neurological Institute is the "OBT" frame (Tipal Instruments, Montréal). This device is physically similar to the Leksell frame ${ }^{6}$ that is in wide use throughout the world. The software system described here is applicable to both frames.

\section{THE FRAME}

Figure 1 shows the stereotactic frame prior to an MRI study. It remains rigidly fixed to the patient throughout the imaging examinations and surgery, although it may be removed and replaced in the same position at a later date in exceptional circumstances. During the imaging session, the frame supports the appropriate fiducial marker plates ${ }^{4}$ described below. During surgery the frame is used to support the surgical instruments and to maintain the reference coordinates used during the imaging procedure.

The frame is constructed from aluminum alloy and plastic materials in such a manner as to provide maximum strength while minimally affecting the quality of the images (avoiding the introduction of artifacts, for example). It consists of a lower ring on which four support posts are mounted and a series of bars at the top to complete the structure. This assembly is sufficiently light for the patient to be able to accommodate it without discomfort and strong enough to support a variety of surgical instruments during surgery. The sides of the frame are engraved with millimeter scales to allow accurate positioning of the instruments during surgery.

\section{FIDUCIAL MARKERS: CT AND MRI}

To enable the coordinates in an image to be accurately calculated, the scaling and positions of two-dimensional slices within the threedimensional frame must be determined. To

From McConnell Brain Imaging Center, Montréal Neurological Institute, PQ; and ELEKTA Instrument AB, Stockholm.

Address reprint requests to T.M. Peters, McConnell Brain Imaging Center, Montréal Neurological Institute, $380 I$ University St, Montréal, $P Q H 3 A-2 B 4$, Canada.

(C) 1989 by W.B. Saunders Company.

0897-1889/89/0202-0005\$03.00-0 


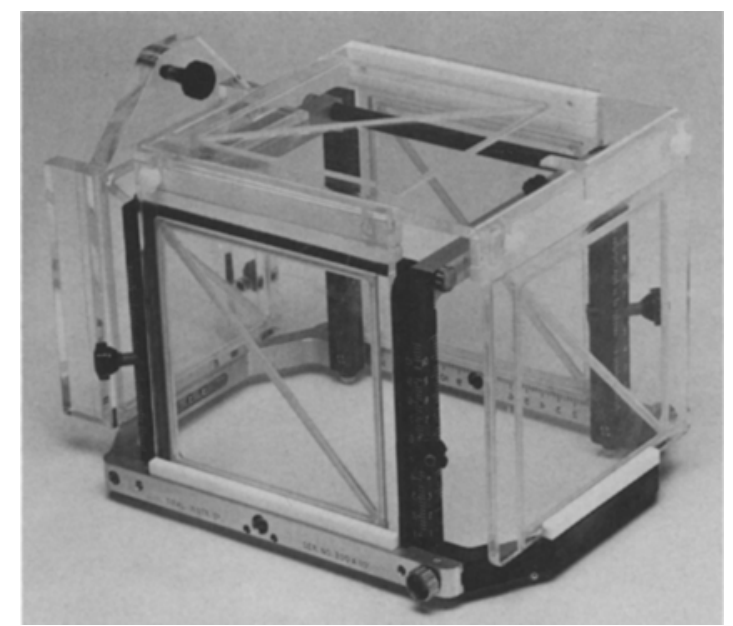

Fig 1. Stereotactic frame with MAI marker plate set in place.

achieve this we surround the frame with a series of plastic plates in which are embedded rods in the shape of a $Z$ or $N$ pattern. Any crosssectional slice through the brain and the frame (including the marker plates) will display three sets of three points surrounding the image. Analysis of the positions of these points in the images, combined with the knowledge of their frame coordinates, enables the location and scale of the slice within the three-dimensional frame of reference to be determined precisely. The threedimensional coordinates of any point within the frame volume may be calculated by analyzing the images in this manner.

The markers must be made from substances that show a strong signal from the modality being used. For this reason, aluminum that has a relatively high radiolucency is used for $\mathrm{CT}$, and a copper-sulphate solution (high NMR signal) enclosed in channels in the plastic plates is used for the markers in MRI. CT scanners make images only in the transverse plane, and so require only three such plates to identify coordinates from a set of images. On the other hand, MRI allows images to be made in sagittal and coronal planes as well as transverse, and so five sets of plates are provided in this case as shown in Fig 1. Stereotactic CT and MR scans are shown in Figs 2 and 3. The markers are clearly evident in the images at the top and sides. The CT image is overlaid with a scale generated by the software package, corresponding to the markings on the frame. Sagittal and coronal images may be recorded with the

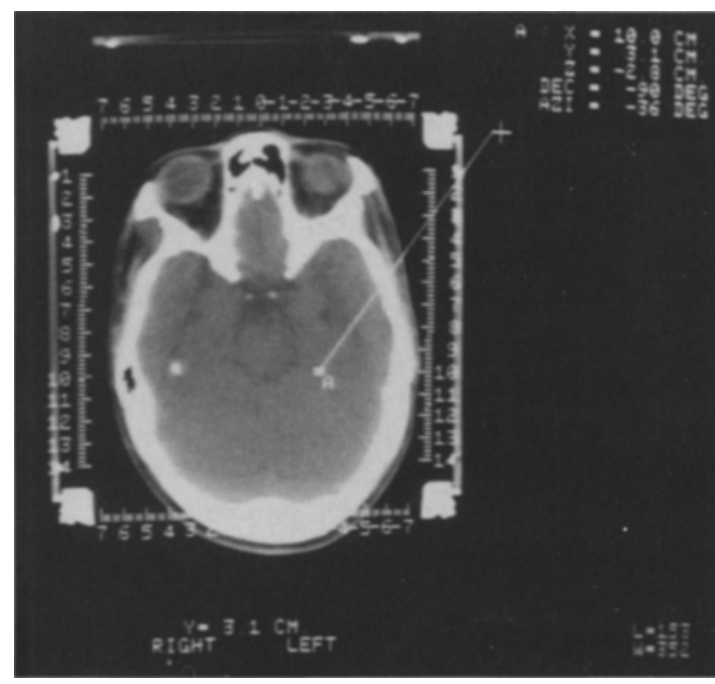

Fig 2. CT image showing fiducial markers, overlaid scale and a trajectory to a potential target point.

MRI system and may be analyzed (subject to the limitations discussed below) in the same manner as transverse images. These other views may be generated from the series of CT images by standard reformatting techniques.

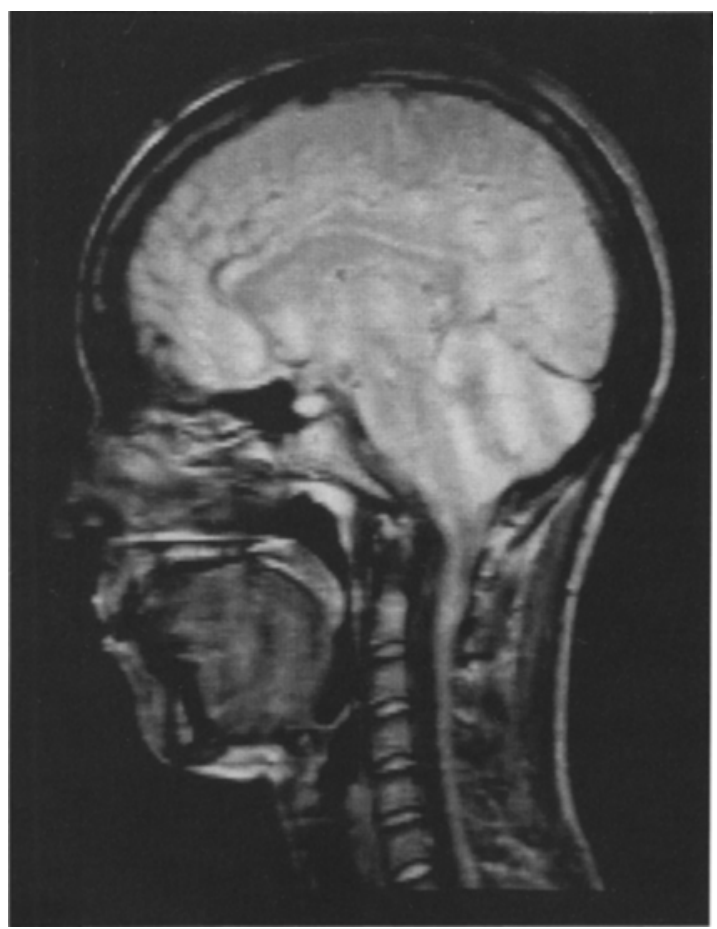

Fig 3. Sagittal MR image showing fiducial markers. 


\section{FIDUCIAL MARKERS: DSA}

Since the DSA technique yields projections of the brain (Fig 4), rather than cross-sections, an approach different to that described above is used. In this case the fiducial markers consist of small metal pellets rather than Z markers embedded in the side plates. Although these points are placed at the corners of similar squares on opposite sides of the frame, they appear in the image (because of differential magnification) to be on squares of different sizes (Fig 5). Nevertheless, these points precisely define the beam geometry of the system. By analyzing images of the same structure in both anterior-posterior and lateral projections, the three-dimensional frame coordinates of structures may be identified from the subtracted angiograms (Appendix).

\section{IMAGE ANALYSIS}

The patient having been scanned with one or more modalities, the images may be analyzed by a software package specially designed for the purpose. We have developed an integrated system based on an IBM-compatible AT personal computer (PC) that enables images from all the modalities to be read from magnetic tape and analyzed in a consistent environment.

The first time each image is accessed, the fiducial markers associated with that image are identified, and the appropriate scaling and positional factors for that slice (or projection in the case of DSA) determined. Target points, trajectories, or volumes may be identified by the operator in any of the images in the data sets, and any

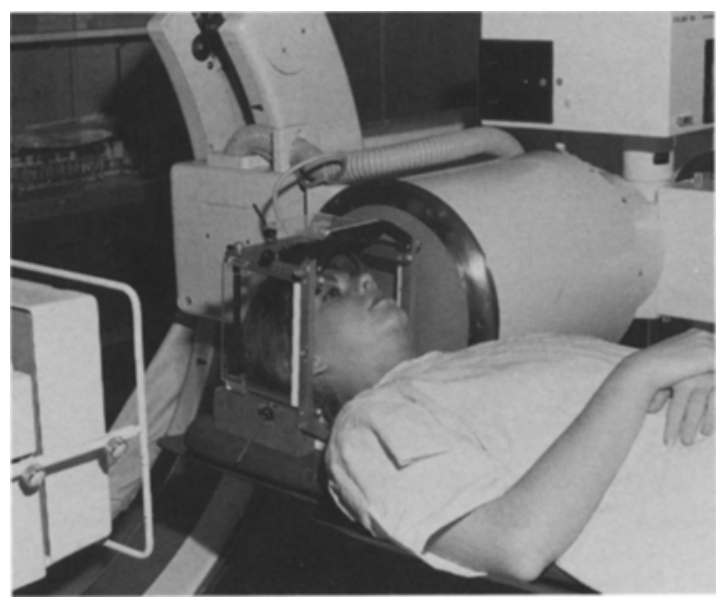

Fig 4. Patient with frame during DSA examination.

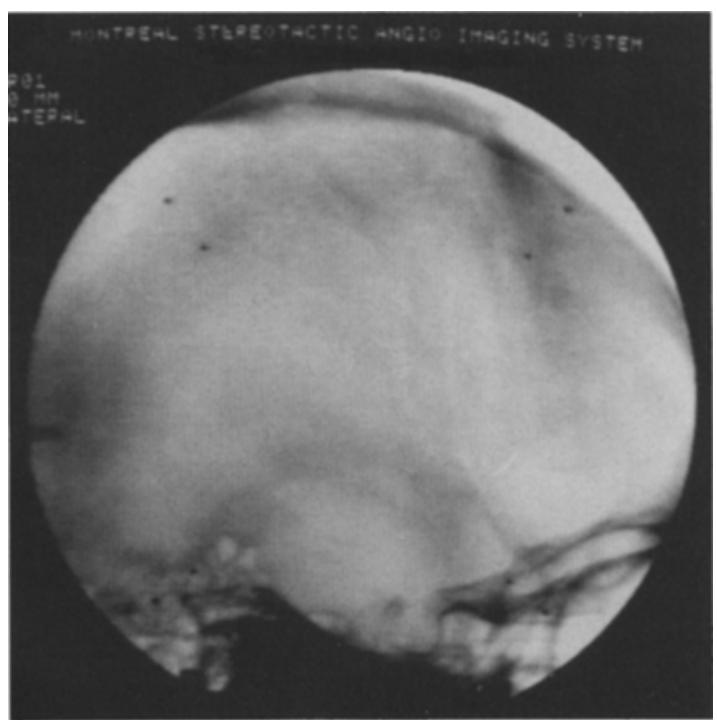

Fig 5. Lateral unsubtracted digital angiogram image. Note the two sets of four markers that the software uses to determine the geometry of the imaging beam.

such targets may immediately be transferred to images obtained from the other modalities.

Target identification in CT or MRI scans is, by a simple linear transformation, based on the known positions of the markers. However, for DSA scans the target must be identified in two orthogonal projections to determine the "third coordinate" of the point.

Target coordinates may be determined on the basis of CT or MRI scans, but the path of approach to these targets is generally planned in conjunction with the DSA images in order to avoid passing through major blood vessles on the way. Figure 2 shows a CT scan with a target and trajectory identified, and Fig 6 shows the pathway as it appears with respect to the subtracted angiogram of the same patient.

\section{COMPUTER SYSTEM}

Other computer systems exist to handle multimodality image data for stereotactic surgery planning, ${ }^{7}$ but most require extensive and expensive hardware facilities. Although our system does not have the capability to display threedimensionally rendered images for example, it nevertheless allows the surgeon to work within a three-dimensional environment. The computer hardware is based on a standard AT-compatible system running MS-DOS. The only additional hardware required is an inexpensive $512 * 512 * 8$ - 


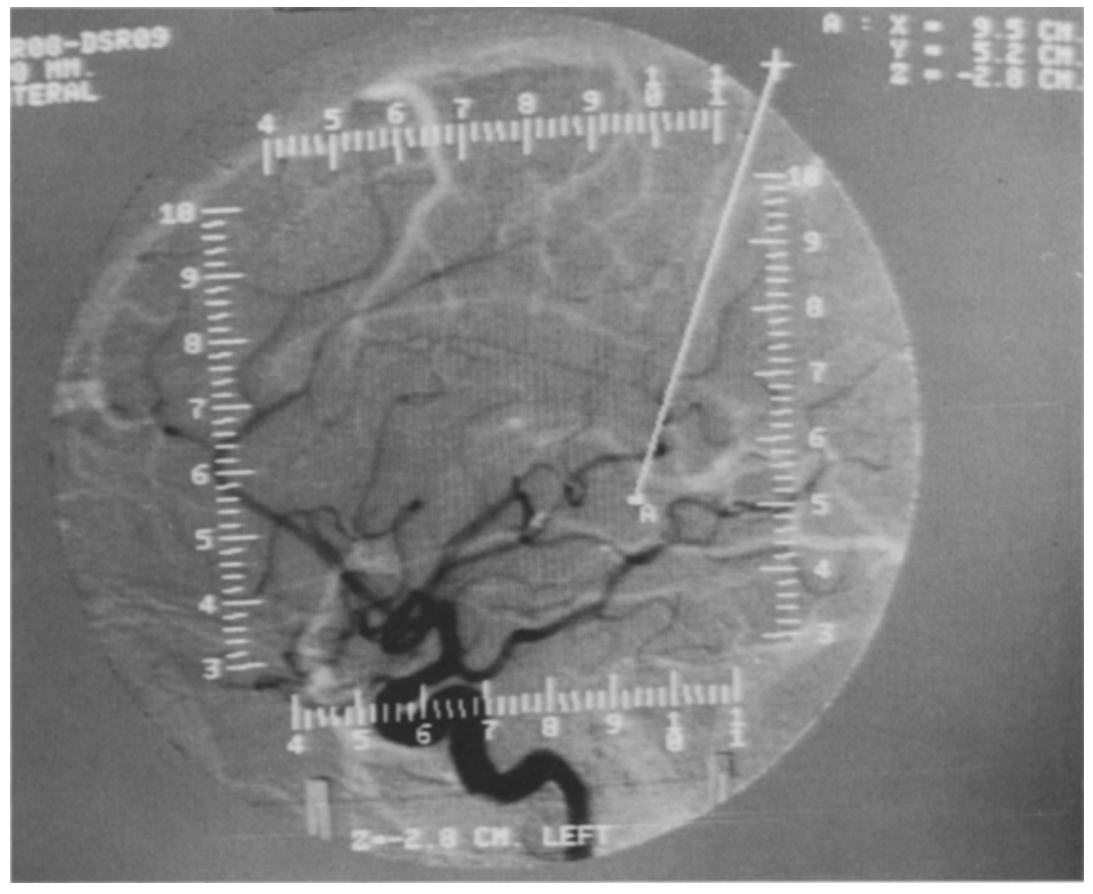

Fig 6. Trajectory marked in Fig 2 as seen on a subtracted lateral DSA image. This image shows the arterial system in black and the venous system in white. bit imaging board and monitor, and an industrystandard 9-track magnetic tape unit. The system uses an 80-Mbyte hard disk. Images are transferred to the system via the magnetic tape unit. The system is compatible with the image formats from most of the popular imaging modalities.

The user interfaces via a mouse that selects menu entries, controls image window and level, and the cursor movement on the screen. The image monitor is an RGB system allowing for display of monochrome or pseudo-color images. In addition, there is a video hard-copy unit for permanent recording of images and analyses generated during the planning session. The entire hardware configuration is shown in Fig 7.

\section{STEREOSCOPIC IMPLEMENTATION}

The apparatus described above has been in use routinely for approximately 2 years. However, we have recently implemented the DSA analysis section on a system capable of displaying stereoscopic images. At the Montréal Neurological Institute, radiologists and surgeons have for many years viewed angiograms stereoscopically by using a stereoscope or viewing the images with crossed eyes, and so there is already a great deal of familiarity with the technique locally.
To record the stereoscopic image pairs, we use a Siemens Angioskop equipped with a stereoscopic tube. The focal spot pair is separated by 25 $\mathrm{mm}$ that gives a stereo separation of $1.5^{\circ}$ at the image receptor. If a greater stereo shift is desired, two sets of images are acquired by angulating the gantry by up to $15^{\circ}$ between runs.

The stereoscopic image pair is viewed on a Tektronix SGA system (Tektronix, Inc., Beaverton, OR) that comprises a liquid-crystal-polarizing plate that may switch the circular polar-

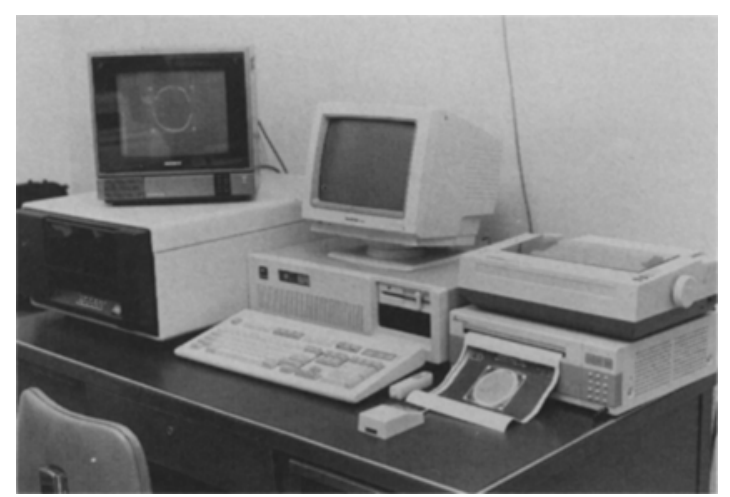

Fig 7. The PC-based imaging system. The tepe read unit is seen at the left, while the video hard-copy unit is on the right. 
ization of transmitted light between right- and left-handed senses in synchrony with the $120 \mathrm{~Hz}$ vertical sync signal of the image display. The viewer observes the image by looking at the screen with the appropriately polarized glasses over each eye. We note that this system can be viewed as a computerized version of the "floatingline" stereoscopic analysis proposed by Cloutier et al. ${ }^{8}$ Most of the software facilities present on the "production" work station are also available on the stereoscopic system. When viewing the images in stereo, it is no longer necessary to look at the anterior-posterior and lateral images to determine the three-dimensional coordinates; the operator merely places the three-dimensional cursor within the volume at the appropriate target point and reads off the coordinates directly. More important, the operator may interactively position a vector representing the trajectory of a probe to the target point, in threedimensional space, to achieve a safe path that avoids traversing any major blood vessels. Note that the accuracy with which the depth coordinate may be determined is a function of pixel size, stereoscopic image disparity, and the ability of the human observer to place a cursor accurately at the desired depth. For this reason it may still be necessary to use the orthogonal view of the vessels to accurately "tie down" the third coordinate. A stereoscopic pair of images depicting a probe placed in the three-dimensional volume is shown in Fig 8 . These may be viewed in stereo by crossing ones eyes and fusing the images.

\section{CLINICAL APPLICATIONS}

During the last 4 years, several hundred patients have been subjected to stereotactic surgical procedures based on the image analysis performed on the system described here. These operations include the implantation of depth electrodes, ${ }^{9}$ and tumor biopsy. In addition to the straightforward stereotactic localization function, an adaptation of the same package handles the dose-planning for stereotactic radiosurgery. After the target volume has been identified, the entire treatment procedure may be simulated..$^{10,11}$ Figure 9 shows the resulting dose plan for a radiosurgery procedure designed to deliver a single high dose of radiation to an arteriovenous malformation in order to ablate the lesion.

\section{ACCURACY}

We have demonstrated a localization accuracy of $\pm 1 \mathrm{~mm}$ when the images are geometrically accurate. This goal has been achieved in our institute with CT, DSA, and transverse MR images. We have found that the gometric reliabil-
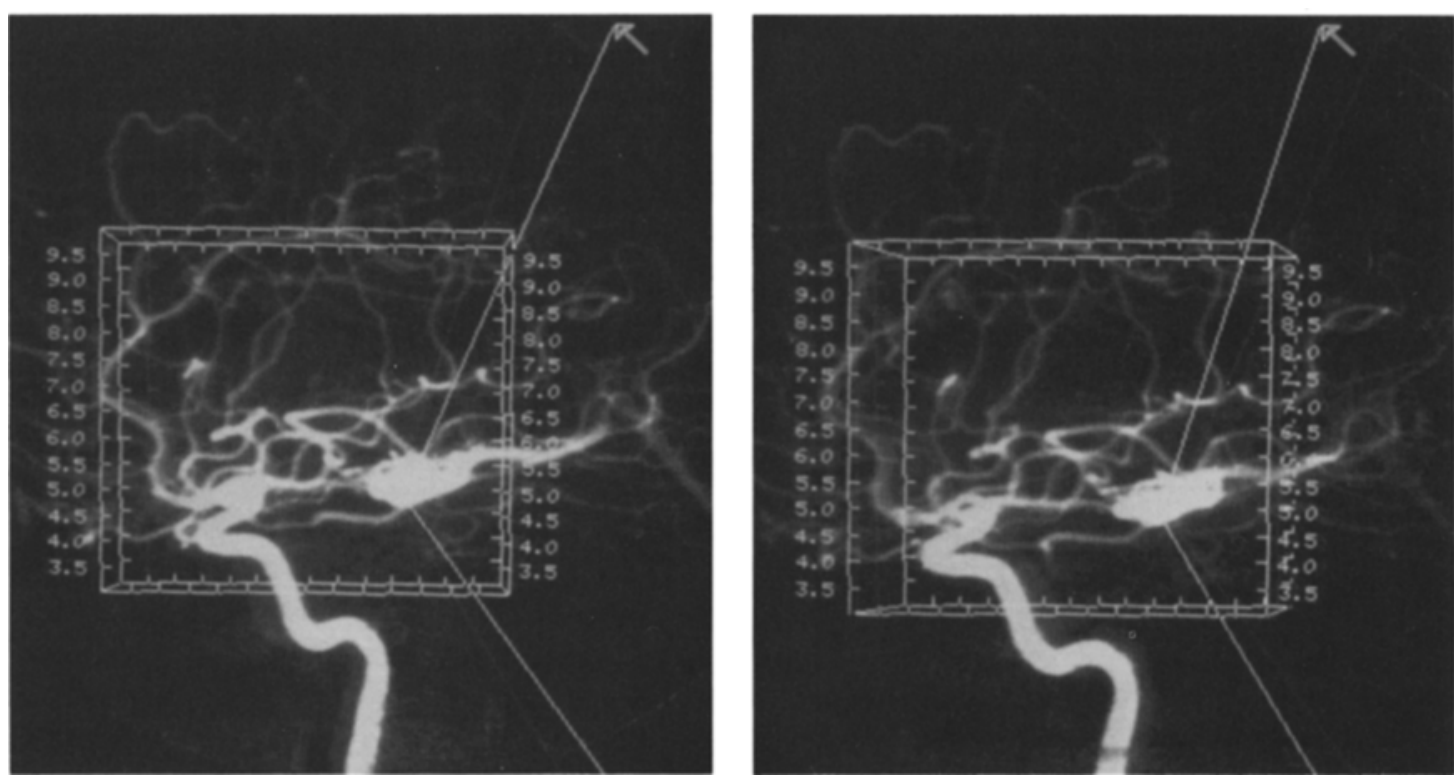

Fig 8. Stereoscopic DSA image pair showing probe placed interactively in the imaging volume. 


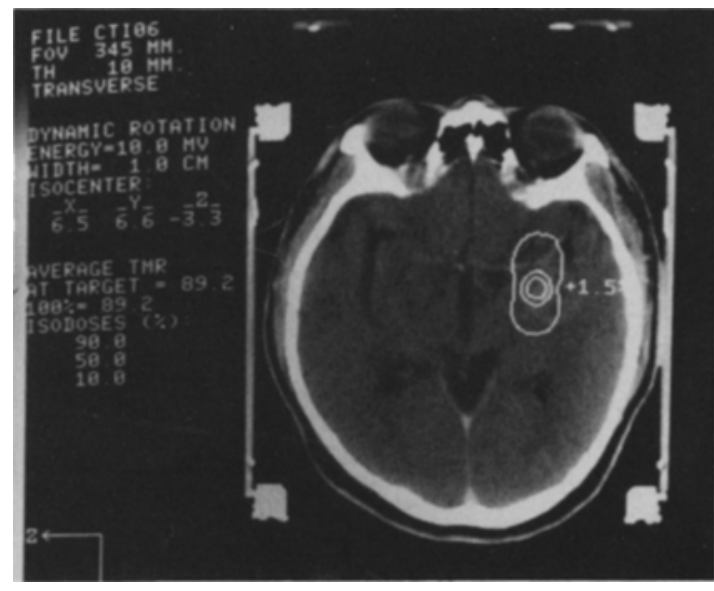

Fig 9. Dose plan for stereotactic radiosurgery procedure.

ity in sagittal and coronal MR images is poor. Part of this problem can be attributed to the alloy components of the metal parts of the frame; but even using a completely plastic frame we found inconsistencies of up to several millimeters when the readout gradient was in the axial direction. This problem indicates a departure from linearity of the gradient fields, and may be eddycurrent-related. Table 1 details the results of the accuracy measurements made using the three modalities and physical measurement to localize the positions of 16 randomly placed pellets within the frame. Table 1 gives the mean error and the standard deviation of this error when the image coordinates were compared with the coordinates that were physically measured within the frame.
Since the frame is graduated in $2-\mathrm{mm}$ steps, we estimate that the errors in the physical measurements are at least as great as the errors introduced by the digitization of the image data. The pixel size for the MR images is $1.27 \mathrm{~mm}$, for the CT images $0.5 \mathrm{~mm}$, and for the DSA images 0.3 $\mathrm{mm}$ (170 $\mathrm{mm}$ field of view). The slice thickness was $3 \mathrm{~mm}$ for CT and $5 \mathrm{~mm}$ for MR. We note that the geometric nonuniformities caused by the lack of homogeneity in the main field may be partially overcome by increasing the band width of the received signal (by increasing the strength of the readout gradient). This parameter may be adjusted in some systems by selecting a minimum "water-fat shift" in the image.

Because of the variability of the MRI geometrical distortion with the imaging pulse sequence selected, we did not consider using a threedimensional warping technique to correct for this problem.

The positions of the fiducial markers are identified in the image by searching for the centroid of the pixels in the neighborhood of the spots in the image. In this manner the center of each fiducial point may be determined to sub-pixel accuracy.

\section{CONCLUSIONS}

We have described an inexpensive but effective PC-AT-based work station for the display and analysis of multimodality images for stereotactic surgery planning. We have discussed the application of the system in routine operation as well as

Table 1. Accuracy of Measurements Using Planning System

\begin{tabular}{|c|c|c|c|c|}
\hline \multirow[b]{2}{*}{ Imaging Tech } & \multicolumn{4}{|c|}{ Error in Each Coordinate (Mean \pm SD) } \\
\hline & $\Delta X(\mathrm{~mm})$ & $\Delta Y(\mathrm{~mm})$ & $\Delta \mathrm{Z}(\mathrm{mm})$ & Dist \\
\hline CT transverse & $-0.4 \pm 0.5$ & $-0.2 \pm 1.1$ & $0.4 \pm 0.6$ & $0.6 \pm 1.3$ \\
\hline DSA & $-0.4 \pm 0.8$ & $-0.7 \pm 1.1$ & $0.3 \pm 0.1$ & $0.8 \pm 1.6$ \\
\hline MRI transverse ap readout & $-0.7 \pm 1.1$ & $-0.1 \pm 2.7$ & $0.1 \pm 1.0$ & $0.6 \pm 2.7$ \\
\hline MRI transverse Ir readout & $-0.8 \pm 0.9$ & $-0.8 \pm 1.6$ & $0.3 \pm 0.8$ & $1.1 \pm 1.9$ \\
\hline MRI sagittal cc readout & $-2.4 \pm 1.3$ & $-0.1 \pm 0.8$ & $1.7 \pm 1.4$ & $2.5 \pm 1.7$ \\
\hline MRI coronal cc readout & $0.5 \pm 1.7$ & $-0.5 \pm 0.6$ & $0.6 \pm 1.2$ & $0.9 \pm 2.1$ \\
\hline MRI sagittal ap readout & $-0.4 \pm 0.8$ & $3.4 \pm 1.3$ & $2.3 \pm 2.5$ & $4.0 \pm 2.8$ \\
\hline MRI coronal Ir readout & $-.35 \pm 1.4$ & $0.7 \pm 0.7$ & $0.1 \pm 0.8$ & $0.8 \pm 1.7$ \\
\hline
\end{tabular}

This table presents the errors in coordinate measurements of test pellets in the stereotactic phantom. Sixteen-point objects were distributed uniformly throughout the volume defined by the frame. The first three entries in each row give the actual measured error in each coordinate, while the last combines these to give a linear distance error. In the table the $X, Y$, and $Z$ coordinates represent the ap, cc, and Ir patient directions, respectively. Note also that the standard deviation of the error is always greatest in the slice-thickness direction for each imaging modality as would be expected.

Abbreviations: ap, anterior-posterior; Ir, left-right; cc, cranio-caudal. 
the extension of the original ideas to include the analysis of steroscopic angiograms, and the planning of stereotactic radiosurgery. We note that while with care we may approach the desired goal of accuracy of $\pm 1 \mathrm{~mm}$ in each coordinate with all modalities, it may not be possible to achieve this level of accuracy with some scanning orientations with MRI without further geometric distortion correction.

\section{APPENDIX}

For every DSA image a minimum of six fiducial markers must be identified in order to identify subsequently the coordinates of structures from the images. Since the position of each identified marker is known precisely in frame coordinates, a series of simultaneous linear equations may be solved using least-squares minimization techniques to yield the 12 elements of a $4 \times 3$ homogeneous transformation matrix. ${ }^{11}$ This matrix provides the transformation between a 3-D frame coordinate point and its displayed position:

$$
[x, y, z, 1] \times\left[\begin{array}{lll}
T_{11} & T_{12} & T_{13} \\
T_{21} & T_{22} & T_{23} \\
T_{31} & T_{32} & T_{33} \\
T_{41} & T_{42} & T_{43}
\end{array}\right]=\omega[U, V, 1]
$$

where: $x, y, z$ are the frame coordinates of the point; $T_{i j}$ are the elements of the homogeneous matrix; $\omega$ is a constant; $U, V$ are screen coordinates of the displayed (projected) point. Note that the perspective scaling is included implicitly within the $x, y, z, \omega$ homogeneous formulation.

These equations may be solved to obtain two equations relating the point's $x, y, z$ coordinates and the displayed (screen) position $U, V$. If the frame coordinates $x, y, z$ of a point are known, then its displayed position $U, V$ can be found. If $U$ and $V$ are known on two single views, then an overdetermined set of equations results allowing us to compute the frame coordinates using a least-squares technique. From a stereoscopic pair of images, these equations may be solved in real time allowing a cursor to be moved around in threedimensional space with its frame coordinates instantly displayed.

\section{REFERENCES}

1. Brown RA: A computerized tomography-computer graphics approach to stereotaxic localization. J Neurosurgery 50:715-720, 1979

2. Bergström M, Grietz T: Stereotactic computed tomography. Am J Roentg 127:167-170, 1976

3. Kelly P: Future possibilities in stereotactic surgery: Where are we going. Appl Neurophysiol 50:1-8, 1987

4. Peters TM, Clark JA, Olivier A, et al: Integrated stereotaxic imaging with CT, MR Imaging and digital subtraction angiography. Radiology 161:821-826, 1986

5. Lundsford LD, Martinez AJ, Latchaw RE: Stereotaxic surgery with a magnetic resonance- and computerized tomography-compatible system. J Neurosurgery 64:872-878, 1986

6. Leksell L, Lindquist C, Adler J, et al: A new fixation device for the Leksell stereotactic system. J Neurosurg 66:626-629, 1987
7. Kall B: The impact of computer and imaging technology in sterotactic surgery. Appl Neurophysiol 50:9-22, 1987

8. Cloutier L, Nguyen $D$, Labissonière, et al: X-ray photogrammetry and floating line concept applied to cerebral probe implantation. Appl Neurophysiol 48:81-82, 1985

9. Olivier A, de Lotbinière A: Stereotectic techniques in epilepsy. Neurosurgery: State of the Art Reviews 2:257-285, 1987

10. Pike B, Podgorsak E, Peters T, et al: Dose distributions in dynamic stereotactic radiosurgery. Med Physiol 14: 780-789, 1987

11. Leksell DG, Stereotactic radiosurgery: Present status and future trends. Neurol Res 9:60-68, 1987

12. Sutherland IE: Three-dimensional data input by tablet. Proc IEEE 62:453-471, 1974 